\title{
Consistent methodology for calculating surface and interface energies
}

\author{
Krzysztof Rapcewicz, Bin Chen, Boris Yakobson, and J. Bernholc \\ Department of Physics, North Carolina State University, Raleigh, North Carolina 27695-8202
}

(Received 20 December 1996; revised manuscript received 1 December 1997)

\begin{abstract}
A consistent approach to the calculation of the surface energy valid for all crystal systems is presented. Voronoi polyhedra are introduced and used in conjunction with the energy-density formalism of Chetty and Martin [Phys. Rev. B 45, 6074 (1992); 45, 6089 (1992)] to provide a methodology for the determination of surface energies. The surface energies of the unrelaxed, unreconstructed GaAs (001) and (111) surfaces are calculated as a test. As an example of the application of the formalism to a low symmetry system, the energies of selected (0001) surfaces of the wide-gap semiconductors $\mathrm{GaN}$ and $\mathrm{SiC}$ are determined. [S0163-1829(98)02012-8]
\end{abstract}

\section{INTRODUCTION}

There has been tremendous interest and progress in the technology of crystal growth over the past few decades. ${ }^{1}$ This has made relevant numerous theoretical questions related to crystal growth and the equilibrium behavior of surfaces. Indeed, intense efforts have been made to understand the energetics of such surfaces. ${ }^{2}$ However, in spite of the substantial progress towards an understanding of the physics of surfaces, the surface energies must be calculated with respect to a reference surface, the reference being different for different surfaces except for specific high-symmetry cases. Since the surface energy plays an important role in the determination of the mode of growth, namely, layer, island or layer-plus-island growth, the lack of a procedure for the calculation of the surface energy is unfortunate. Indeed, if meaningful $a b$ initio predictions about the equilibrium crystal shape and preferred growth directions are to be made, it will be necessary to have a database of surface energies of reconstructed surfaces for different directions. This requires that the evaluations of the surface energies be consistent between calculations.

The situation can be summarized as follows: On the basis of general considerations, it can be shown that for crystals of sufficiently high symmetry, the surface energy can be calculated unambiguously. For the remaining systems, it can only be calculated consistently, i.e., the surface energy is defined up to a gauge; this gauge is of no consequence in the determination, for instance, of equilibrium crystal shapes. Practically, however, the surface energy, when it is unambiguously defined, can be calculated absolutely with a slab calculation only when the two surfaces are crystallographically identical; most often, only a relative surface energy can be determined.

Three cases therefore exist: (1) the surface energy is unambiguously defined and can be determined using a slab calculation; the (001) surface of a zinc-blende semiconductor is such a case; (2) the surface energy is unambiguously defined, but slab calculations give only relative surface energies; the (111) surface of a zinc-blende semiconductor serves as a case in point; (3) the surface energy can only be defined consistently (the gauge must be set, once and for all, for the calculations); the (0001) surface of a wurtzite semiconductor is such an example.

Chetty and Martin ${ }^{3-5}$ developed an approach that could be used to calculate the surface energies in the second case above. They introduced the concepts of the energy density and symmetry-adapted unit cell to provide a procedure for the calculation of the surface energy and applied their approach to the GaAs (111) surface and interfaces. However, symmetry-adapted unit cells can be used only if the space group has sufficiently high symmetry, namely, a center of symmetry, two axes of rotation, or an axis of rotation and a mirror plane not through this axis. For the third case, no methodology exists to calculate the surface energy.

We have generalized the energy-density approach of Chetty and Martin ${ }^{3,4}$ to a consistent methodology for the calculation of surface energies for all systems including those of case three above. In this approach, the slab and bulk are considered to be built from blocks whose shape is determined by the symmetry of the bulk crystal. The "energy cost" of each block is evaluated and the "cost" of creating a surface, the surface energy, is obtained by summing up the cost of each block in the slab and subtracting the costs of the equivalent blocks in the bulk. The shape of the blocks is determined according to a geometric rule; thus the approach provides a consistent way of determining the surface energy that can be used for low-symmetry systems. Further, the symmetric nature of the blocks ensures that symmetryequivalent surfaces will have the same surface energy and hence the methodology will reproduce the results of slab calculations, and those of Chetty and Martin. The blocks can be chosen to be either neutral or charged, the former being our preferred choice because they are more intuitive.

The paper is organized as follows: a brief résumé of the surface energy begins the presentation, after which the standard calculations of the surface energy are discussed. In addition, a conceptual approach using only total-energy calculations and scaling arguments, which is applicable to systems of sufficiently high symmetry, is presented. This approach clarifies the issues involved in unambiguously defining the surface energy, but it is not feasible at present. The energydensity approach of Chetty and Martin is then reviewed together with the implementation of the energy-density formalism using symmetry-adapted unit cells. A generalization of the energy-density formalism using Voronoi polyhedra (VP) is introduced and applied to GaAs (001), GaAs (111), SiC (0001), and GaN (0001). The latter two cases provide examples of low-symmetry systems in which symmetry- 
adapted unit cells cannot be used. The paper concludes with a summary.

\section{SURFACE ENERGY}

In this section we review the theory of the surface energy and the role that symmetry plays in the definition of the surface energy. In particular, for systems that are of low symmetry the surface energy cannot be defined absolutely but only up to a gauge, which must be the same for all directions.

Consider a system containing two phases, 1 and 2, and a dividing surface. The excess energy $E^{(s)}$ due to the presence of the dividing surface is the (excess) quantity defined through the relation

$$
E=E^{(1)}+E^{(2)}+E^{(s)},
$$

where $E$ is the total energy of the system and $E^{(1)}\left(E^{(2)}\right)$ is the energy of the equivalent bulk system of phase 1 (2). The definitions for other surface thermodynamic quantities, such as the entropy, $S^{(s)}$, etc. are similar. ${ }^{6}$ The surface free energy per unit area is defined to be

$$
\gamma=e^{(s)}-T s^{(s)}-\sum_{i} \mu_{i} \Gamma_{i},
$$

where small letters denote the corresponding excess quantities per unit area with the exception of $\Gamma_{i}=N_{i}^{(s)} / A$, which is the excess number of particles of the $i$ th species per unit area, and $\mu_{i}$ is the chemical potential of the $i$ th species.

The surface free energy defined in this way measures the work required to create a new surface. It is, in general, distinct from the work needed to deform a surface that is the surface stress. In fluids, as is well known, the surface free energy is isotropic and is the same as the surface stress. ${ }^{6}$ In contrast, the surface free energy in solids is generally anisotropic, which is to say that the surface free energy, $\gamma(\hat{\mathbf{n}})$, is a function of the direction of the outward-pointing normal. Further, the surface free energy and the surface stress are distinct quantities.

The surface free energy per unit area is important in the determination of the equilibrium geometry of small crystals and the equilibrium shape of small particles in contact with a substrate. Under conditions of constant temperature $T$, volume $V$, and chemical potentials $\mu_{i}$, the excess free energy of the system due to the presence of the surface is

$$
\Psi=\int d A \gamma(\hat{\mathbf{n}}),
$$

where $d A$ is the element of surface area with outwardpointing normal $\hat{\mathbf{n}}$. The equilibrium shape thus minimizes the excess free energy.

For a single crystal at constant temperature and chemical potential, the minimization of Eq. (3) subject to the constraint of fixed volume leads to the Wulff construction for determining the equilibrium shape, ${ }^{6}$ while a modification of the Wulff construction, the Winterbottom construction, ${ }^{7}$ gives the shape of a crystal for a given orientation of the substrate and, thus, information about the growth mode.
Since the surface energy manifests itself physically through the minimization of the integral in Eq. (3), it is possible to add to the surface energy a gauge term $\mathbf{C} \cdot \hat{\mathbf{n}}$ (where $\mathbf{C}$ is a constant vector) without changing the equilibrium shape. ${ }^{8,9}$ Symmetry, however, permits a nonzero $\mathbf{C}$ for only 10 point groups, namely, $C_{6}, C_{6 v}$ (which includes wurtzite), $C_{4}, C_{4 v}, C_{3}, C_{3 v}, C_{2}, C_{2 v}, C_{1 h}$, and $C_{1}$. For $C_{1}, \mathbf{C}$ is completely arbitrary; for the rest, symmetry constrains the choice of $\mathbf{C}$. For $C_{1 h}, \mathbf{C}$ must lie on the mirror plane; for the remaining eight point groups, $\mathbf{C}$ must lie along the unique axis. In other words, one, two, or three arbitrary scalars must be chosen. ${ }^{9}$

\section{DETERMINATION OF THE SURFACE ENERGY FROM TOTAL ENERGY CALCULATIONS}

For the surface energy two situations exist: (i) for high symmetry systems, the surface energy is unambiguously defined (combining cases one and two of the introduction); (ii) for low symmetry systems, the surface energy is defined up to a gauge. In this section, we begin by pointing out that slab calculations permit a determination of the absolute energy only in high-symmetry cases. In other cases, slab calculations provide only relative surface energies, even if the surface energy is absolutely defined. We then present a method that permits the calculation of absolute surface energies using polyhedra and scaling behavior.

The calculations employed to determine the surface energy for a given crystal direction are generally slab calculations. The slab consists of a finite number of layers and is made infinite in the plane of the surface through the imposition of periodic boundary conditions. The need to decouple the two surfaces from one another dictates the thickness of the slab and also the number of vacuum layers (the calculations are usually carried out using supercells). We define the energy of the equivalent bulk as the sum of the number of atoms of each species times the chemical potential of that species. Invariably, a single configuration is used to determine the total ground state energy of the slab at $T=0$. In a multicomponent system, the chemical potential for a single species is not defined for a single configuration. In fact, the chemical potentials that occur in the slab calculations are external parameters and can take arbitrary values; however, physical arguments are invoked to establish limits upon the variability of the chemical potentials of the individual species.

At equilibrium, the chemical potential of a species is equal in all phases that are in contact. This observation can be exploited to impose constraints on the possible equilibrium values. In particular, it is generally assumed that the bulk is in equilibrium with the surface, i.e., that the sum of the chemical potentials of the individual species, $\mu_{A}, \mu_{B}$, etc. equals the bulk chemical potential $\mu_{A B \cdots Z \text { (bulk) }}$,

$$
\mu_{A B \cdots Z \text { (bulk })}=\mu_{A}+\mu_{B}+\cdots+\mu_{Z} .
$$

Further, the chemical potential for a given species cannot be above the chemical potential of its elemental bulk phase, since the bulk phase would then be unstable with respect to precipitation of the elemental bulk. Thus, the maximum value of the chemical potential of a given species, say $A$, is equal to the chemical potential of its elemental bulk phase 
$\mu_{A \text { (bulk) }}$. The heat of formation $\Delta H_{f}$, which relates the chemical potential in the elemental bulk system to the chemical potential in the compound is defined by

$$
\mu_{A B \cdots Z(\text { bulk })}=\mu_{A(\text { bulk })}+\mu_{B(\text { bulk })}+\cdots+\mu_{Z(\text { bulk })}-\Delta H_{f} .
$$

It can be used to set bounds for the chemical potential of the individual species in the bulk, viz.

$$
\mu_{\alpha(\text { bulk })}-\Delta H_{f} \leqslant \mu_{\alpha} \leqslant \mu_{\alpha(\text { bulk })},
$$

where $\alpha=A, B, \ldots, Z{ }^{10}$ It is worth emphasizing that these bounds only delineate the range over which the bulk is stable. If the chemical potential falls outside of these bounds, the bulk will become metastable and kinetics will determine the subsequent behavior of the system, i.e., whether a change of phase will occur, whether the metastable phase will be very long lived, etc. Thus the bounds Eq. (6) provide a reasonable range over which to consider the surface energy. ${ }^{11}$

For certain directions of sufficiently high symmetry, the calculation of the surface energy is simplified. Along these special directions, it is possible to find a slab that has identical surfaces. More explicitly, the surfaces are related by a reflection about the center of the slab and, possibly, by a rotation in the plane of the surface. For a slab of material $A B \cdots Z$ consisting of $N_{A}$ atoms of species $A, N_{B}$ atoms of species $B$, etc., in a sufficiently simple structure [the (001) surface is an example of such a surface]; the surface energy is

$$
\gamma=\frac{1}{2}\left(E_{\text {slab }}-N_{A} \mu_{A}-N_{B} \mu_{B}-\ldots N_{Z} \mu_{Z}\right),
$$

where $E_{\text {slab }}$ is the total energy of the slab. ${ }^{12}$ Along lower symmetry directions, the slab calculations give only the surface energy relative to some reference, usually taken to be the $1 \times 1$ unreconstructed, unrelaxed surface.

It is possible, at least in principle, to determine the absolute surface energies for the symmetry directions for which slab calculations yield only relative surface energies provided the crystal has a space group of sufficiently high symmetry. Construct a polyhedron with identical faces: the total energy of a polyhedron, $E_{\text {poly }}$, that has $n_{f}$ identical faces, $n_{e}$ edges, and $n_{v}$ vertices, and contains $N_{A}$, atoms of species $A$, $N_{B}$ atoms of species $B$, etc., is

$$
\begin{aligned}
E_{\text {poly }}= & N_{A} \mu_{A}+N_{B} \mu_{B} \cdots+N_{z} \mu_{Z}+\alpha_{f} n_{f} E_{\text {surf }} L^{2}+\alpha_{e} n_{e} E_{\text {edge }} L \\
& +\alpha_{v} n_{v} E_{\text {vertex }},
\end{aligned}
$$

where $E_{\text {bulk }}$ is the energy per unit volume of the bulk material comprising the polyhedron, $E_{\text {surf }}$ is the surface energy per unit area, $E_{\text {edge }}$ is the edge energy per unit length, $E_{\text {vertex }}$ is the energy of a vertex, and $L$ is the characteristic size of the polyhedron. The $\alpha$ coefficients depend upon the detailed shapes of the surfaces, edges and vertices but the different scaling behavior of each of the energy terms can be exploited to calculate the surface energy. After subtracting out the bulk energy, the remainder consists of surface terms, which vary as $L^{2}$, edge terms, which vary as $L$, and vertex terms, which are independent of $L$. For a sufficiently large polyhedron, the contribution of the edge terms to the remainder will be negligible and the surface energy can be calculated to order $1 / L$, viz.

$$
E_{\text {surf }}=\frac{1}{\alpha_{f} n L^{2}}\left(E_{\mathrm{poly}}-N_{A} \mu_{A}-N_{B} \mu_{B} \ldots-N_{z} \mu_{Z}\right)+\mathcal{O}(1 / L)
$$

A two-component system with a zinc-blende lattice provides an example of a crystal with sufficiently high symmetry; a tetrahedron can be constructed that has four faces comprised of (111) surfaces terminated by the same species, A or B. ${ }^{13}$ Clearly this approach is valid only for a limited set of symmetry directions. For the 11 Laue point groups and 11 of the 21 remaining point groups that have either two rotation axes or one rotation axis with a mirror plane not through the axis, it is possible to find a polyhedron whose faces are composed of crystallographically equivalent surfaces. ${ }^{9,13}$

The surface energies for other directions can be calculated by slicing off one of the vertices; the surface energy for all but one of the faces is known and, in the scaling limit, the unknown surface energy can be determined to $\mathcal{O}(1 / L)$. In this case, too, the polyhedron must be sufficiently large to ensure that edge and vertex effects are negligible. Although such calculations are not feasible at present, the procedure described in this section illustrates the existence of unambiguously defined surface energies for crystals with symmetries as described above. For crystals with lower symmetries, an appropriate convention for defining the vector $\mathbf{C}$, the same for all surfaces, must be established. For these systems, the surface energies are measured with respect to a single reference surface.

\section{ENERGY-DENSITY FORMALISM}

The total energy of a charge-neutral system of electrons with density $\rho(\mathbf{r})$ within the density-functional theory is

$$
E=T_{s}[\rho]+V_{H}[\rho]+E_{\mathrm{XC}}[\rho]+V_{i e}[\rho]+E_{i i}\left(\left\{\mathbf{R}_{I}\right\}\right),
$$

where $T_{s}[\rho]$ is the Kohn-Sham kinetic-energy functional, $V_{H}[\rho]$ is the Hartree functional, $E_{\mathrm{XC}}[\rho]$ is the exchangecorrelation functional, $V_{i e}[\rho]$ is the electron-ion energy and $E_{i i}\left(\left\{\mathbf{R}_{I}\right\}\right)$ is the electrostatic self-energy of the atoms located at $\left\{\mathbf{R}_{I}\right\}$. Minimization of this functional subject to the constraint that the number of electrons is fixed leads to the Kohn-Sham equations, the solution of which gives the ground-state energy and density of the charge-neutral system. ${ }^{14,15}$

The energy density can be defined ${ }^{3,4}$ through the relation

$$
E=\int_{V} d^{3} r \mathcal{E}(\mathbf{r})
$$

Consistent with this definition of the energy density, any function $f(\mathbf{r})$ that integrates to zero can be added to the energy density. In principle, this presents a problem for the energy-density formalism; such an arbitrary function, a gauge function, would make the integrals over subvolumes arbitrary. In practice, however, such a problem does not arise. The energy functional and the energy density are constructed with each term having a physical motivation and thus such an arbitrary, unphysical function can be excluded by construction. Notwithstanding this exclusion of arbitrary unphysical gauge terms, there is an intrinsic variability of the energy density in any multicomponent system, which is due 
to the nature of the lattice sum for the Coulomb energy of the ions. This intrinsic variability can be used to incorporate the variability of the externally imposed chemical potential.

The forms of many of the components of the energy density follow in a straightforward manner from the definition, Eq. (11), although their concrete realization depends upon the details of the implementation of the calculation. In particular, the present calculations use a supercell geometry, the pseudopotential formalism and a plane-wave basis. The kinetic-energy density is

$$
\mathcal{T}(\mathbf{r})=\frac{1}{2} \sum_{n} f_{n} \nabla \psi_{n}^{*}(\mathbf{r}) \cdot \nabla \psi_{n}(\mathbf{r})
$$

where the $\left\{\psi_{n}\right\}$ are the Kohn-Sham wave functions and $\left\{f_{n}\right\}$ their occupations. This symmetric form of the kinetic energy is the more basic form that enters into the variational formulation of quantum mechanics. ${ }^{16,17}$ In systems possessing periodic boundary conditions, the minimization of this functional gives the standard Laplacian-form of the kinetic energy in the Kohn-Sham equations. ${ }^{18}$ The use of Bloch's theorem permits the expansion of the Kohn-Sham wave functions, viz.

$$
\mathcal{T}(\mathbf{r})=\frac{1}{2} \sum_{i, \mathbf{k}} f_{i \mathbf{k}} \nabla \psi_{i, \mathbf{k}}^{*}(\mathbf{r}) \cdot \nabla \psi_{i, \mathbf{k}}(\mathbf{r})
$$

where the sum on $\mathbf{k}$ is over the first Brillioun zone. Specializing to a plane-wave basis, it is more efficient, from a computational point of view, to evaluate the gradient of the wave function in reciprocal space and then to Fourier transform into real space, where $\mathcal{T}(\mathbf{r})$ is a point-wise product. In real space, the exchange-correlation energy density is the pointwise product of the electron density and the exchangecorrelation energy per electron of the homogeneous, interacting electron gas taken at the local density, i.e.,

$$
\mathcal{E}_{\mathrm{XC}}(\mathbf{r})=\rho(\mathbf{r}) \varepsilon_{\mathrm{XC}}(\mathbf{r})
$$

The determination of the energy density for the electrostatic terms requires a careful treatment. The reasons are computational and mathematical. The Hartree potential can be most efficiently calculated for a charge density that has zero net charge, while the lattice sums of the Coulomb potentials of the ions must be regularized through the use of techniques related to those introduced by Ewald. The square of the electric field, which is the negative of the gradient of the Hartree potential, i.e.,

$$
\mathbf{E}(\mathbf{r})=-\nabla v_{h}(\mathbf{r})
$$

gives the Maxwell energy density, viz.

$$
\mathcal{E}_{M}(\mathbf{r})=\frac{1}{8 \pi}|\mathbf{E}(\mathbf{r})|^{2}
$$

This term can be most efficiently calculated in a manner similar to the kinetic-energy density. The electric field is first evaluated in reciprocal space and then transformed into real space, where the energy density is calculated as a pointwise product.
The pseudoion-pseudoion energy per supercell must be much more extensively reworked analytically and the sum restricted to the supercell by use of the minimum-imaging convention in order to obtain

$$
E_{\mathrm{ion}}=\frac{1}{2} \sum_{I, J \in \Omega}{ }^{\prime} \frac{Z_{I} Z_{J}}{R_{I J}} \operatorname{erfc}\left[\frac{R_{I J}}{\sqrt{R_{c, I}^{2}+R_{c, J}^{2}}}\right]-\frac{1}{2} \sum_{I \in \Omega} \frac{2 Z_{I}}{\sqrt{2 \pi} R_{c, I}} .
$$

The first term in the pseudoion-pseudoion electrostatic selfenergy is due to the Coulomb interaction between a Gaussian pseudoion at $\mathbf{R}_{I}$ and one at $\mathbf{R}_{J}$, while the second term is the electrostatic self-energy of the charge distribution of the pseudoion at $\mathbf{R}_{I}$. The energy density for this contribution to the total energy is obtained by taking the energy corresponding to an atom at $\mathbf{R}_{I}$ to be the coefficient of a $\delta$ function centered on that atom, viz.

$$
\begin{aligned}
\varepsilon_{\text {ion }}(\mathbf{r})= & \sum_{I, J \in \Omega}{ }^{\prime} \frac{Z_{I} Z_{J}}{R_{I J}} \operatorname{erfc}\left[\frac{R_{I J}}{\sqrt{R_{c, I}^{2}+R_{c, J}^{2}}}\right] \times \alpha_{I J} \delta\left(\mathbf{r}-\mathbf{R}_{I}\right) \\
& -\frac{1}{2} \sum_{I \in \Omega} \frac{2 Z_{I}}{\sqrt{2 \pi} R_{c, I}} \delta\left(\mathbf{r}-\mathbf{R}_{I}\right)
\end{aligned}
$$

where the prime on the sum indicates that $I \neq J$. The unknown coefficients $\alpha_{I J}$ reflect an ambiguity in the resolution of this term into a density; the electrostatic energy between a pseudoion at $\mathbf{R}_{I}$ and one at $\mathbf{R}_{J}$ is a sum evaluated at the ion positions, not an integral evaluated over all space. When the two ions in the sum are distinct, there is no a priori way of assigning a portion of the weights to the contribution of each ion to the sum. The $\alpha_{I J}$ may be chosen to be different for different pairs of species subject only to the constraint

$$
\alpha_{I J}+\alpha_{J I}=1 \text { for } I \neq J \text {. }
$$

Thus in a two-component system there are two additional degrees of freedom, $\alpha_{I J}$ and $\alpha_{J I}$, and one constraint so that, in effect, there is one degree of freedom. For convenience, the $\left\{\alpha_{I J}\right\}$ were set equal to one-half in the calculations.

The local-pseudopotential energy can be written as

$$
E_{\mathrm{lps}}=\sum_{I \in \Omega} \int_{\Omega} d^{3} r \rho(\mathbf{r}) U_{\mathrm{sr}, I}(\mathbf{r}),
$$

where

$$
U_{\mathrm{sr}, I}(\mathbf{r})=U_{\mathrm{lps}, I}(\mathbf{r})-\int_{V} d^{3} x \frac{n_{g, I}(\mathbf{x})}{|\mathbf{r}-\mathbf{x}|}
$$

so that the energy density for the local pseudopotential is

$$
\mathcal{E}_{\mathrm{lps}}(\mathbf{r})=\rho(\mathbf{r}) U_{\mathrm{sr}}(\mathbf{r})
$$

In Eq. (21), $n_{g, I}(\mathbf{x})$ is a Gaussian charge density with integrated charge $Z_{I}$ and width $R_{c, I}$ centered at $\mathbf{R}_{I}$.

The nonlocal pseudopotential is short range in real space. Following Chetty and Martin, ${ }^{3}$ the nonlocal pseudopotentialenergy associated with an ion at $\mathbf{R}_{I}$ is gathered into a $\delta$ function centered on that ion. The smallest volumes that will be considered are much larger than the region over which the nonlocal pseudopotential is nonzero so that no spurious re- 
sults are introduced by this treatment of the nonlocal pseudopotential. The energy density for the nonlocal pseudopotential is

$$
\mathcal{E}_{\text {nlps }}(\mathbf{r})=\sum_{I \in \Omega} \mathcal{E}_{\text {nlps }, I} \delta\left(\mathbf{r}-\mathbf{R}_{I}\right),
$$

where the coefficient of the $\delta$ function is

$$
\mathcal{E}_{\mathrm{nlps}, I}=\sum_{i, \mathbf{k}} f_{i \mathbf{k}} \int_{\Omega} d^{3} r \int_{\Omega} d^{3} r^{\prime} \psi_{i, \mathbf{k}}^{*}(\mathbf{r}) \hat{U}_{\mathrm{nlps}, I}\left(\mathbf{r}, \mathbf{r}^{\prime}\right) \psi_{i, \mathbf{k}}\left(\mathbf{r}^{\prime}\right) .
$$

Thus $\mathcal{E}(\mathbf{r})$ is a sum of densities, viz.

$$
\mathcal{E}(\mathbf{r})=\mathcal{E}_{\text {kin }}(\mathbf{r})+\mathcal{E}_{\mathrm{XC}}(\mathbf{r})+\mathcal{E}_{M}(\mathbf{r})+\mathcal{E}_{\mathrm{ps}}(\mathbf{r})+\mathcal{E}_{\text {ion }}(\mathbf{r}),
$$

where $\mathcal{E}_{\text {kin }}(\mathbf{r})$ is the kinetic-energy density, $\mathcal{E}_{\mathrm{XC}}(\mathbf{r})$ is the exchange-correlation energy density, $\mathcal{E}_{M}(\mathbf{r})$ is the Maxwell energy density (a particular form of the energy density for the Hartree term), $\mathcal{E}_{\mathrm{ps}}(\mathbf{r})$ is the pseudopotential energy density, and $\mathcal{E}_{\text {ion }}(\mathbf{r})$ is the ion-ion electrostatic self-energy density.

\section{EVALUATION OF THE SURFACE ENERGY}

The present approach to the calculation of the surface energy necessarily involves two parts: the energy-density methodology $y^{3,4}$ and an integration procedure. This approach extends the method developed by Chetty and Martin ${ }^{3-5}$ by generalizing the integration method so that it can be used even in systems of low symmetry.

\section{A. Surface energy using symmetry-adapted cells}

The approach of Chetty and Martin ${ }^{3-5}$ makes use of symmetry-adapted unit cells for the integration. The boundaries of a symmetry-adapted unit cell are symmetry planes of the crystal. Therefore, the integral of the energy density over this unit cell is gauge independent and the number of atoms in the cell can be unambiguously determined. The surface energy is then

$$
\sigma=\int_{V_{s}} d^{3} r \mathcal{E}_{\text {slab }}(\mathbf{r})-\sum_{i} N_{i} \mu_{i},
$$

where $V_{s}$ is the volume of the symmetry-adapted unit cell and $N_{i}$ is the number of atoms of the $i$ th species inside the cell with $i=A, B, \ldots, Z$. The externally imposed chemical potential of the $i$ th species is $\mu_{i}$; their sum is subject to the constraint, Eq. (4). This approach can be employed to calculate the surface energy of those directions in which the surface is cut obliquely by these symmetry planes. Chetty and Martin ${ }^{4}$ applied their method to the (100) and (111) surfaces of GaAs. The (111) surfaces of a zinc-blende crystal are cut obliquely by the (100) and the (110) planes. However, this approach cannot be used for a crystal whose point group is one of the 10 point groups for which the absolute surface energy is not defined, i.e., there will be surfaces that are not cut obliquely by a sufficient number of symmetry planes. Wurtzite (0001) is an example of such a surface; as the result of the hexagonal symmetry, the appropriate symmetryadapted cell cannot be defined.

\section{B. The Voronoi polyhedron}

The integration procedure must permit a consistent evaluation of the surface energy for those low-symmetry systems for which the absolute surface energy cannot be defined. Such an approach is needed because, in general, it is necessary to treat the two surfaces of the slab in supercell calculations differently. For instance, to minimize the charge transfer between surfaces, one of the surfaces may need to passivated and consequently it is desirable to have a methodology that permits the surface energy of each face to be calculated separately. Further, in order to make meaningful $a b$ initio predictions about equilibrium crystal shape and preferred growth directions, a database of surface energies of reconstructed surfaces for different directions must be made. This requires that the evaluations of the surface energies be consistent between calculations.

In the approach that we have developed, we view the bulk and the slab as built of blocks. These blocks are constructed according to a well-defined rule, which is a natural generalization of the definition of the familiar Wigner-Seitz cell. The same rule is used to obtain the Voronoi polyhedra in the slab: deep in the slab, where the bulk is recovered, the Voronoi polyhedra are identical to those of the bulk; near the surface, they are deformed. With each block we associate an energy, namely, the integral of the energy density over that volume. The "total cost" of constructing a slab and the equivalent bulk is determined by simply adding up the respective energies associated with all of the Voronoi polyhedra. The surface energy is then simply the difference in "cost"' between the portion of the slab containing one surface and the equivalent bulk.

In a one-component crystalline system condensed on a lattice without a basis, the integral of the energy density over any volume that is charge neutral is unique; it is the total energy per particle. The arbitrariness of the choice of volume notwithstanding, there is a geometrically motivated volume, namely, the Wigner-Seitz cell. The Wigner-Seitz cell possesses the symmetry of the lattice, is space filling and charge neutral. If the crystal has a basis of two identical atoms, then there is a difference between the positions of the atoms making up the crystal and the lattice points. The Wigner-Seitz cell for this crystal is the set of all points closer to a given lattice point than to all other lattice points. As for the case of a lattice without a basis, the Wigner-Seitz cell for the lattice with a two-atom basis is charge neutral, possesses the symmetry of the point group of the lattice and is space filling. However, it contains two atoms. The Wigner-Seitz cell can be generalized to a Voronoi polyhedron about each atom. This polyhedron, which is space filling and charge neutral, is invariant under the largest point subgroup of the space group of the lattice.

Consider a lattice that has a basis of two distinct atoms. As in the case of a lattice with a basis of two identical atoms, the Wigner-Seitz-like volume for a given atom will have the symmetry of the largest point group that is a subgroup of the space group of the crystal. The union of such volumes with the similarly defined cells for the other atom of the basis will be space filling, but the volume need not be charge neutral. We will generally choose to use a Voronoi polyhedron that has these properties and, because it is most intuitive, is charge neutral. This Voronoi polyhedron is obtained if the 
faces are translated inward or outward along its normal until the volume is charge neutral. If the normal to a face lies along the vector joining two identical atoms, the face will not be shifted. Only a face that has its normal pointing towards an atom of a different species than the one at the center of the cell will shift. However, it is also possible to use noncharge-neutral polyhedra. Indeed, in strongly ionic systems where the charge exchange between the cation and anion is significant or in $\mathrm{C}_{60}$ where the carbon atoms are not identi$\mathrm{cal}$, it is necessary to use charged Voronoi polyhedra. Because the role of the Voronoi polyhedra is to provide a way of counting atoms and the energies (chemical potentials) associated with them, it is not necessary to add terms to the Hamiltonian when using charged Voronoi polyhedra. Mathematical aspects of the definition of the Voronoi polyhedron are discussed in Appendix I.

The integral of the energy density over the Voronoi polyhedron, $\Omega_{\mathrm{vp}}$, in bulk is

$$
E^{*}=\int_{\Omega_{\mathrm{vp}}} d^{3} r \mathcal{E}(\mathbf{r})
$$

and will be referred to as the bulk-atom energy. In a one component system, $E^{*}$ is equal to the chemical potential. The integral of the energy density over the Voronoi polyhedron for species $A$ will be referred to as the bulk-atom energy of species $A$, etc.

For a multicomponent system, the bulk-atom energy satisfies the constraint of equilibrium

$$
\mu_{A B \cdots Z}=E_{A}^{*}+E_{B}^{*}+\cdots+E_{Z}^{*},
$$

where $\mu_{A B} \cdots Z$ is the chemical potential. The total energy of a system of $N_{A}$ atoms of species $A$ and $N_{B}$ atoms of species $B$, etc., is

$$
E_{\mathrm{tot}}=N_{A} E_{A}^{*}+N_{B} E_{B}^{*}+\cdots+N_{Z} E_{Z}^{*} .
$$

The bulk-atom energy plays a role identical to the imposed chemical potential in a slab calculation, namely, of an externally imposed parameter (cf. Sec. II). It can assume arbitrary values in the bulk system subject only to the constraint of Eq. (28). The same physical constraints that are used to delineate the possible values of the chemical potential also delimit the range of the bulk-atom energy.

For atoms deep inside of a slab where the bulk has been recovered, the Voronoi polyhedra of the slab are identical to the bulk Voronoi polyhedra. The shape of each Voronoi polyhedron depends upon the locations of the neighboring atoms. Consequently, as the surface is approached and atoms are displaced from their bulk positions, the volumes will deform. Because the atoms on the surface do not have any neighbors in the vacuum, the volumes for atoms at the surface will extend out to infinity, cf. the definition in Appendix I and Fig. 1.

As an example, consider diamond. In a diamond crystal, which has space group $F d 3 m$, the Voronoi polyhedron is a snub tetrahedron ${ }^{19,20}$ that has sixteen vertices and sixteen faces. The snub-tetrahedron is invariant under the point group $T_{d}$ and is pristine, i.e., it is space filling only if it has ideal proportions. ${ }^{20}$ Its four hexagonal faces lie between the nearest neighbors and the atom at the origin, while each of

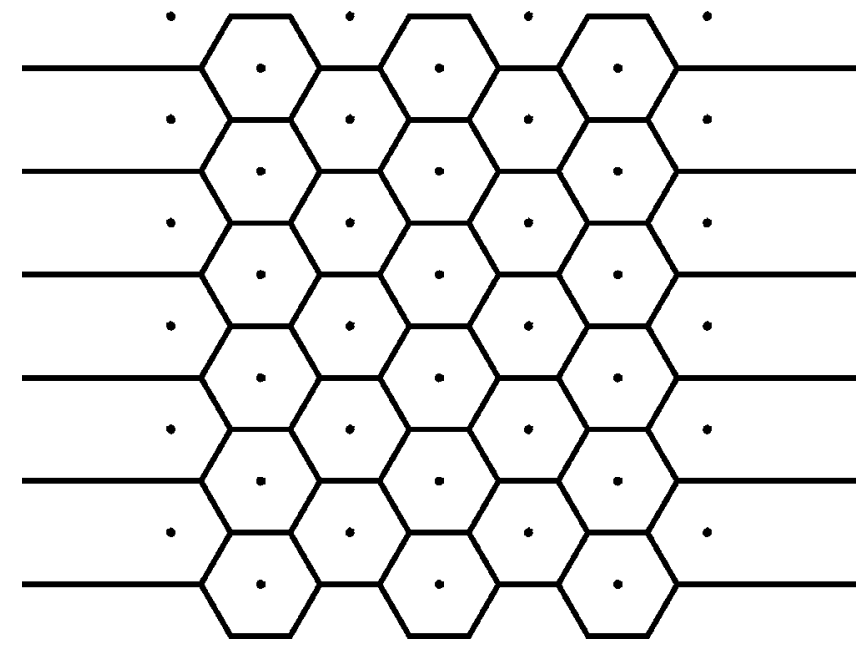

FIG. 1. A two-dimensional slab showing the atomic cells. In the center of the slab, the bulk Voronoi polyhedra are recovered; the cells at the surface extend to infinity.

its twelve isosceles triangular faces is a bisecting plane between the atom at the center and a next-nearest neighbor. The zinc-blende crystal structure for a compound semiconductor $A B$ has space group $F \overline{4} 3 \mathrm{~m}$. In all but the exceptional case of equal weights for the two species, the Voronoi polyhedra will be deformed snub tetrahedra, i.e., snub tetrahedra with nonideal proportions (the union of the two deformed snub tetrahedra-one for $A$ and the other for $B-i s$ space filling). In GaAs, the snub tetrahedra are very close to pristine.

\section{Surface energy using Voronoi polyhedra}

The use of Voronoi polyhedra permits the determination of the deviation from bulk behavior in a straightforward manner. For each atom, there is one polyhedron; deep in the slab, the Voronoi polyhedra of the bulk are recovered. A section of the slab that extends from a point deep in the slab out to a point deep in the vacuum and contains $N_{A}$ atoms of species $A, N_{B}$ atoms of species $B$, etc., has a total energy equal to the sum of the energy of each of the Voronoi polyhedra contained in that portion of the slab, i.e.,

$$
E_{\mathrm{sec}}=\sum_{I} \int_{\Omega_{I}} d^{3} r \mathcal{E}_{\mathrm{slab}}(\mathbf{r}),
$$

where $\Omega_{I}$ is the Voronoi polyhedron for an atom at $\mathbf{R}_{I}$ and $I$ runs over all atoms in the section of the slab. In order to obtain the surface energy, the atoms of the slab can be put into a one-to-one correspondence with the atoms of the bulk. This equivalent bulk is comprised of the appropriate bulk, Voronoi polyhedra. Its total energy is simply

$E_{\mathrm{bulk}}=\sum_{I} \int_{\Omega_{V P, I}} d^{3} r \mathcal{E}_{\mathrm{bulk}}(\mathbf{r})=N_{A} E_{A}^{*}+N_{B} E_{B}^{*}+\cdots+N_{Z} E_{Z}^{*}$,

where $\Omega_{V P, I}$ is the Voronoi polyhedron for an atom at $\mathbf{R}_{I}$ and $I$ runs over all atoms in the equivalent bulk [cf. Eq. (29)]. The surface energy is thus 


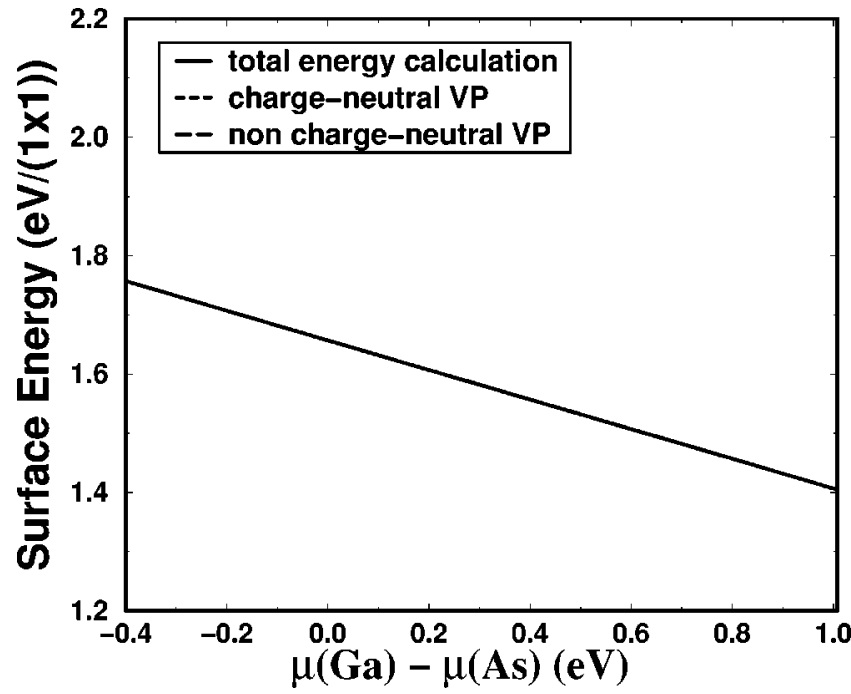

FIG. 2. Comparison of the surface energy of the galliumterminated surface of GaAs (001) from a total-energy calculation with the values obtained using the energy-density formalism with charge-neutral and charged Voronoi polyhedra [the charge of the Voronoi polyhedron (VP) centered on the gallium atom is 3.09e]. The results agree to less than $0.01 \mathrm{eV}$. The linear dependence upon the gallium chemical potential predicted from the formalism is -0.50 , in excellent agreement with the theoretical result of -0.50 (the linear dependence upon the difference chemical potential is $-0.25)$. The range of the abscissa is chosen, by convention, to be about twice the heat of formation. In this and the following figures, it has been slightly broadened to include the possibility of metastability as discussed in the text (Sec. II).

$$
\begin{aligned}
E_{\mathrm{surf}}\left(E_{I}^{*}\right) & =E_{\mathrm{sec}}-E_{\mathrm{bulk}} \\
& =\sum_{I}\left[\int_{\Omega_{I}} d^{3} r \mathcal{E}_{\mathrm{slab}}(\mathbf{r})-E_{I}^{*}\right]
\end{aligned}
$$

where the sum on $I$ runs over all atoms in the section of the slab.

It should be emphasized that the Voronoi polyhedra provide a way of counting atoms and the energies (bulk-atom energies) associated with them. In particular, the energy density is calculated for the entire cell and not as the superposition of energy densities obtained by using the charge density in each Voronoi polyhedron. For instance, the Maxwell energy density, Eq. (16), is calculated using the density in the entire cell; Voronoi polyhedra are not used. Thus problems related to long-range multipole moments do not arise.

Finally, in order to effectively use the formalism, the slab must be sufficiently thick to ensure that the bulk is recovered in the center of the slab. In the example shown in Fig. 2, 9.5 layers were used in order to obtain an accuracy of better than $0.01 \mathrm{eV}$.

\section{Integrals over the Voronoi polyhedra}

Numerous algorithms for the construction of Voronoi polyhedra exist. ${ }^{21-26}$ The particular algorithm we followed is similar to that of Finney. ${ }^{22}$ A subset of neighbors is selected and the vertices of the Voronoi polyhedron are then determined. The list of vertices thus generated is sorted and ver- tices that differ by less than a tolerance or that fall outside of a cutoff are rejected. As a check to verify that the polyhedra fill the entire space, the total volume is calculated as a sum of the individual polyhedra and compared with the total volume of the supercell. In all cases the agreement was excellent, indicating that the polyhedra so generated were space filling.

The charge density and the energy density, defined on a grid, must be integrated over the convex Voronoi polyhedra. Due to the role played by Brillouin-zone integrations in electronic-structure calculations, there has been constant interest in the development of techniques for the evaluation of Brillouin-zone integrations. ${ }^{27-30}$ The integrals of the energy and charge density over the Voronoi polyhedra are the realspace equivalent to the Brillouin-zone integration.

A careful treatment of contributions to the integral from the boundary is essential to the accurate numerical evaluation of integrals over polyhedra such as occur in the present case. The approach that was used was to decompose the polyhedron into tetrahedra by a Delaunay triangulation. ${ }^{31}$ This, however, requires vertices on the surface and edges of the polyhedron. These vertices were obtained by intersecting the lines and planes of the fast fourier transform (FFT) grid with the surface and edges of the polyhedron, respectively. A Delaunay triangulation of this lattice, comprised of the FFT grid and the vertices on the faces and edges, was carried out using GEOMPACK. ${ }^{32}$ The values of the functions on the surface of the polyhedron and at the center of gravity of the tetrahedra were calculated using a tricubic interpolation. The integration rule for the tetrahedron was of third order. ${ }^{33}$ In this way sufficient integration accuracy for the charge and energy in a Voronoi polyhedron was obtained.

\section{APPLICATIONS}

The present approach was used to calculate the surface energies of the (001) and (111) unreconstructed, unrelaxed surfaces of GaAs and of the unreconstructed, unrelaxed surfaces and a few reconstructions of the (0001) surfaces of wurtzite $\mathrm{SiC}$ and $\mathrm{GaN}$. In each case, the energy-density formalism with Voronoi polyhedra was used to calculate the reference surface energy of the unrelaxed, unreconstructed surfaces.

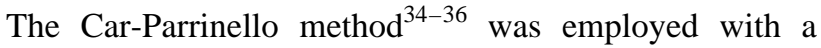
plane-wave cutoff of $14 \mathrm{Ry}$ for GaAs and $30 \mathrm{Ry}$ for $\mathrm{SiC}$ and $\mathrm{GaN}$. The Perdew-Zunger parametrization of the exchangecorrelation energy ${ }^{37,38}$ was chosen. Norm-conserving pseudopotentials ${ }^{39-41}$ were used for gallium, arsenic, carbon, hydrogen, and silicon, while the nitrogen pseudopotential was an optimized soft-core pseudopotential of $\mathrm{Li}$ and Rabii. $^{42}$ The pseudopotentials were $p$ local pseudopotentials, except for the gallium pseudopotential, which was $d$ local. The non-local potentials were employed using the KleinmanBylander approach. ${ }^{43}$ For GaAs, the gallium pseudopotential included the nonlinear core correction. ${ }^{44,45}$

A supercell was used to reimpose the translational invariance that is broken along the direction parallel to the surface normal. For the calculations involving the (001) galliumterminated surface, the $2 \times 2$ supercell contained 9.5 bilayers of GaAs (both faces gallium terminated) and 2.5 bilayers of vacuum; for the calculations of the (111) surfaces, the $2 \times 2$ supercell contained six bilayers of GaAs and three bilayers 
of vacuum. A $2 \times 4$ supercell containing five bilayers of material and three bilayers of vacuum was used for $\mathrm{SiC}$; the $2 \times 2 \mathrm{GaN}$ supercell contained six bilayers of material and four bilayers of vacuum.

In order to minimize charge transfer between the faces, hydrogen atoms passivated one face of the $\mathrm{GaN}$ and $\mathrm{SiC}$ slabs. This was successful as charge transfer was found to be small.

If the work functions of the two surfaces are different, then the periodic boundary conditions of the supercell enforce a common electrostatic potential in the vacuum and result in an unphysical change in the electric potential in the vacuum region equal to the difference between the two work functions. The field, induced by this change, was cancelled by adding a dipole layer in the vacuum region.

\section{A. Surface energy of GaAs (001)}

The calculation of the surface energy of the (001) surface of GaAs provides a good test of the present formalism. In this case a slab in which both surfaces have the same termination exists. The surface energy can thus be calculated using the total-energy approach and the surface energies of the two surfaces can be independently calculated using the energy-density approach presented herein. Figure 2 shows the gallium surface energy calculated with the total-energy method compared to that obtained with the energy-density formalism with Voronoi polyhedra for two different cases: (1) charge-neutral Voronoi polyhedra and (2) charged Voronoi polyhedra in which the polyhedra around the gallium atoms have charge $3.09 e$, while those centered on the arsenic atoms have charge 4.91e. Only the calculations of one face of the slab are shown. Similar results are obtained for the other face.

The surface energies agree to better than $0.01 \mathrm{eV}$ over the entire range. In all of the cases, the linear dependence of the surface energy upon the gallium chemical potential has a slope of -0.50 , which is in very good agreement with the exact value of -0.50 . The value of this slope is not assumed in the calculation, but is a result that confirms the correctness of the approach. Thus it is possible to use either neutral or charged Voronoi polyhedra.

\section{B. Surface energy of GaAs (111)}

The surface energies for the (111) Ga- and As-terminated faces are shown in Fig. 3. As expected, a linear dependence upon the gallium chemical potential is observed with a slope of -0.27 for the gallium-terminated surface and 0.26 for the arsenic-terminated surface. This should be compared with the analytical results of -0.25 for the Ga-terminated surface and 0.25 for the As-terminated surface. The energy of the As-terminated face is lower than that of the Ga-terminated face over the entire range and the numerical results are in good agreement with those of Chetty and Martin.

\section{SiC surface energy}

The surface energies for selected reconstructions ${ }^{46}$ of the $\mathrm{Si}$ - and C-terminated faces are shown in Fig. 4. The unrelaxed, unreconstructed surfaces are observed to depend linearly on the chemical potential of silicon. In both cases the

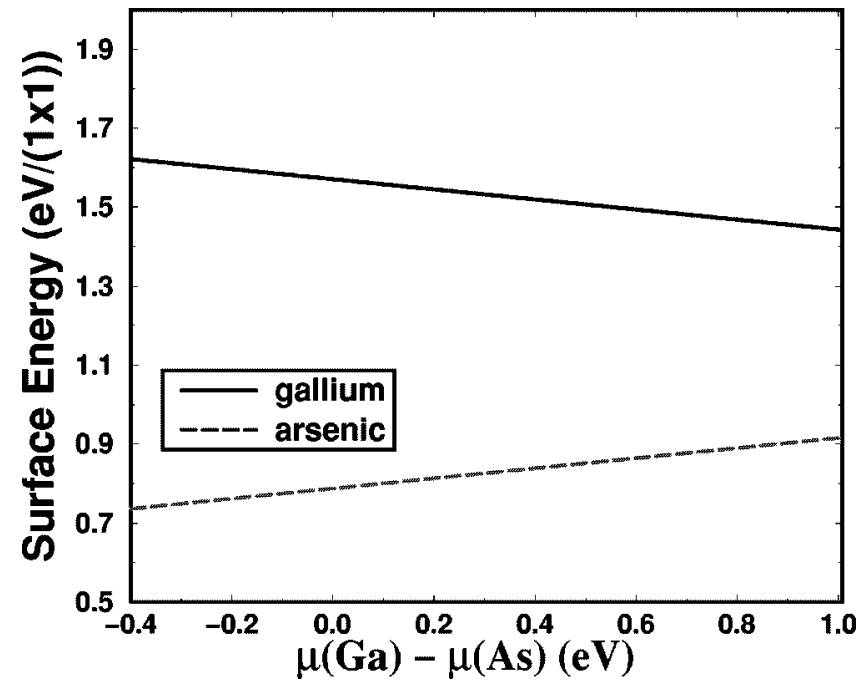

FIG. 3. Surface energy of the unrelaxed, unreconstructed GaAs(111) surfaces calculated using the energy-density formalism with Voronoi polyhedra.

$2 \times 2 \pi$-bonded reconstruction has the lowest energy of the reconstructions considered. It should be noted, however, that lower-energy reconstructions exist and are currently being studied.

\section{GaN surface energy}

The surface energies for the Ga- and N-terminated faces are shown in Fig. 5. With $\Gamma$-point sampling we find that on the gallium-terminated surface the nitrogen-adatom and gallium-vacancy structures have the lowest energies over the entire physical range; the energy difference is smaller than the accuracy of the calculation. On the nitrogen-terminated surface, the nitrogen vacancy has the lowest energy while the gallium adatom has a somewhat higher energy. Although the energies of the individual surfaces can be shifted by a constant (cf. Sec. I), the sum of the surface energies is an absolutely defined quantity.

\section{SUMMARY AND CONCLUSIONS}

The surface energy plays a critical role in determining equilibrium crystal shapes and modes of growth. The standard $a b$ initio method for the evaluation of surface energies employs total-energy slab calculations. These calculations give the absolute surface energies only for those exceptional directions in which the two faces of the slab are crystallographically the same. For other surfaces, they give the energies relative to some reference surface, even in those cases for which the absolute surface energy exists. In order to evaluate the surface energies of lower-symmetry surfaces (for which the absolute surface energy is defined) Chetty and Martin ${ }^{3-5}$ introduced the energy-density formalism together with symmetry-adapted unit cells; however, the energydensity approach procedure is not applicable to systems where such cells cannot be defined.

The surface energy, which is an excess thermodynamic quantity, is absolutely defined for crystals that have a center of symmetry, or two axes of rotation or an axis of rotation and a mirror plane not through this axis. For lower-symmetry 

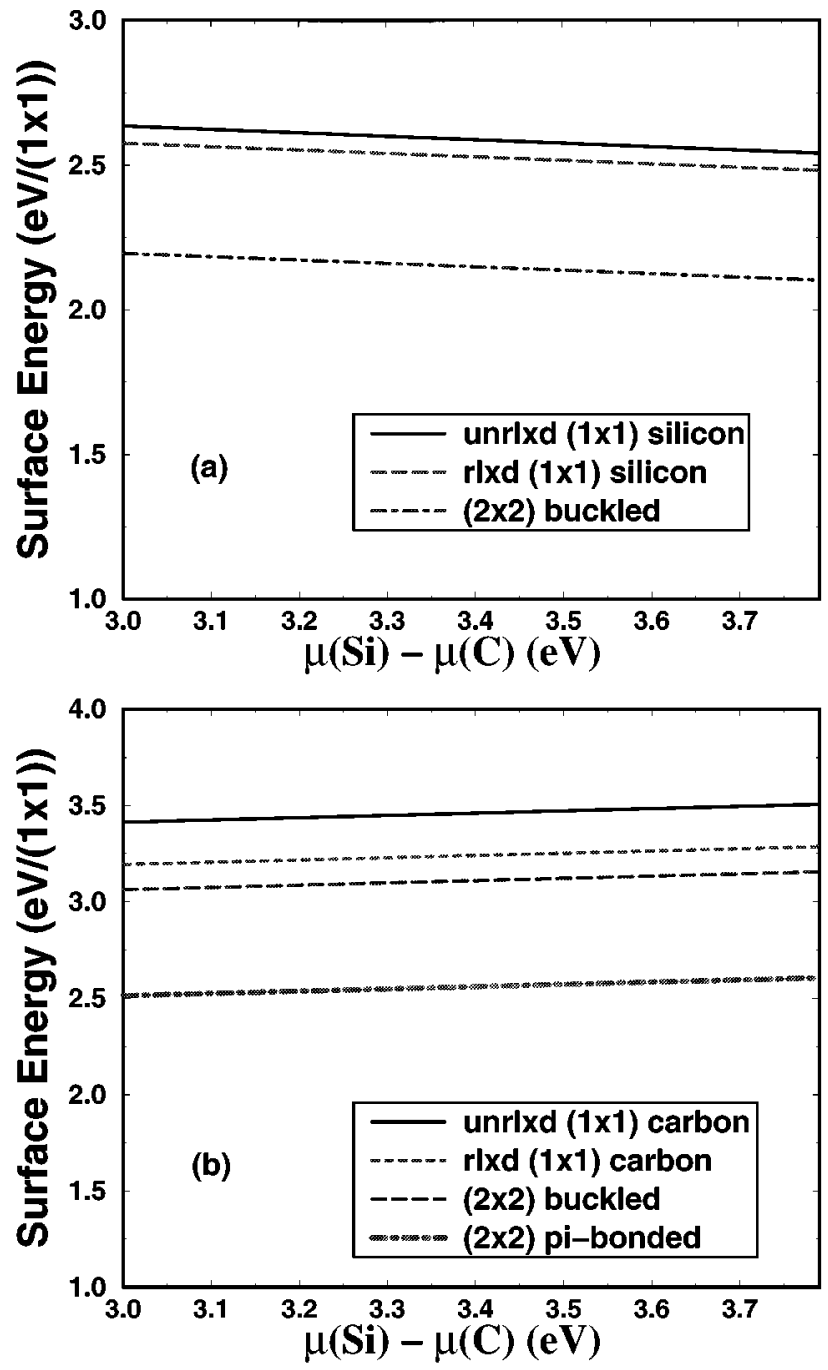

FIG. 4. Surface energy of selected reconstructions of $\mathrm{SiC}$ (0001) calculated using the energy-density formalism with Voronoi polyhedra: (a) the silicon-terminated surface and (b) the carbonterminated surface. The linear dependence of the surface energies of the reconstructions of the carbon-terminated surface on the carbon chemical potential has slope -0.24 ; the linear dependence of the surface energies of the reconstructions of the silicon-terminated surface on the silicon chemical potential has slope -0.24 .

crystals, the surface energy is defined up to a gauge term $\mathbf{C} \cdot \hat{\mathbf{n}}$, where $\mathbf{C}$ is constrained by symmetry for all but the point group $C_{1}$; hence for these systems the surface energy must be defined in a consistent manner for all directions. ${ }^{8,9}$

We have developed a generalization of their approach that permits the evaluation of surface energies in all cases, subject only to the physical ambiguity of the gauge term $\mathbf{C} \cdot \hat{\mathbf{n}}$. Our method uses appropriately defined Voronoi polyhedra to calculate the differences between the bulk and surface contributions to the integrated energy density, thus isolating the excess energy introduced by the surface.

This approach was used to calculate the surface energy of gallium-terminated GaAs (001). Since the surface energy in this case can be evaluated using a total-energy calculation, it provides a good test of the method. The surface energies of the two approaches (total-energy and energy-density with Voronoi polyhedra) agreed to less than $0.1 \mathrm{eV}$ per surface
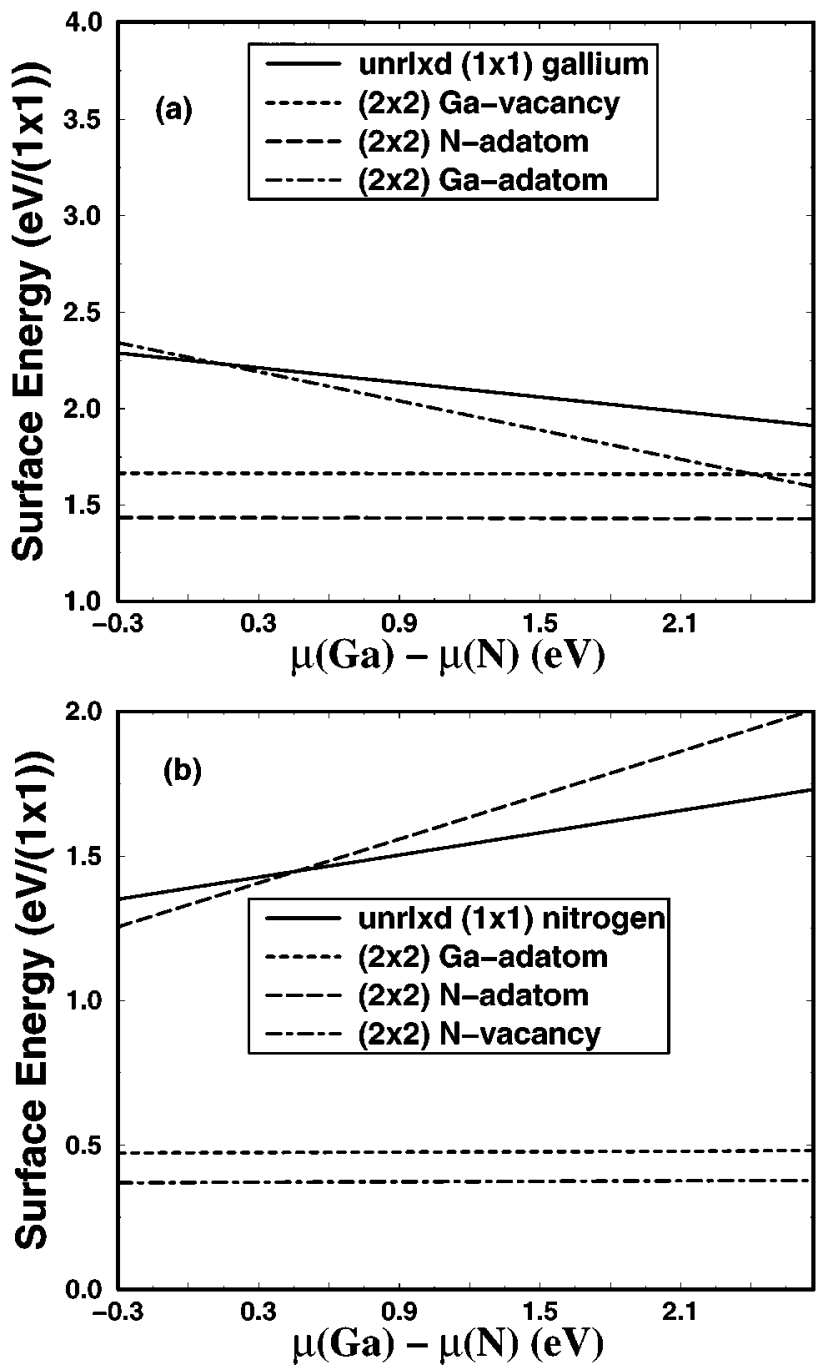

FIG. 5. Surface energy of the reconstructions of GaN (0001) calculated using the energy-density formalism with Voronoi polyhedra: (a) the gallium-terminated surface and (b) the nitrogenterminated surface. For the unreconstructed, unrelaxed galliumterminated face, the surface energy depends linearly upon the gallium chemical potential with slope of -0.25 ; for the unreconstructed, unrelaxed nitrogen-terminated face, the surface energy depends linearly upon the nitrogen chemical potential with slope of -0.25 .

atom. The surface energies of the gallium- and arsenicterminated faces of GaAs (111) were determined and found to be in agreement with those of Chetty and Martin. ${ }^{4}$ Finally, the approach was used to evaluate the surface energies of selected reconstructions of the (0001) surfaces of the widegap semiconductors $\mathrm{SiC}$ and $\mathrm{GaN}$.

\section{ACKNOWLEDGMENTS}

The authors acknowledge the contributions of Dr. Y. G. Hwang and Dr. P. Bogusławski during the early part of this work.

\section{APPENDIX: MATHEMATICAL DETAILS CONCERNING THE VORONOI POLYHEDRON}

For a Bravais lattice which has lattice points $\left\{\mathbf{x}_{j}\right\}$, the region of space nearer to the lattice point $\mathbf{x}_{i}$ than to $\mathbf{x}_{j}$ (the 
so-called dominance region of $\mathbf{x}_{i}$ over $\mathbf{x}_{j}$ ) is the half-space

$$
D\left(\mathbf{x}_{i}, \mathbf{x}_{j}\right)=\left\{\mathbf{x}|| \mathbf{x}-\left.\mathbf{x}_{i}\right|^{2}<\left|\mathbf{x}-\mathbf{x}_{j}\right|^{2}\right\} ;
$$

the boundary of this half-space is the plane defined by

$$
\left|\mathbf{x}-\mathbf{x}_{i}\right|^{2}=\left|\mathbf{x}-\mathbf{x}_{j}\right|^{2}
$$

and the Wigner-Seitz cell is the intersection of all such halfspaces, i.e.,

$$
V\left(\mathbf{x}_{i}\right)=\bigcap_{j \neq i} D\left(\mathbf{x}_{i}, \mathbf{x}_{j}\right) .
$$

Since the Wigner-Seitz cell is the intersection of convex half-spaces, it is convex. Because its faces are planes, it is a polyhedron.

The isogonal point group of a crystal is the group formed from all of the point-group operations which occur in the space group. ${ }^{47}$ If the space group is symmorphic, the isogonal point group will be a subgroup of the space group; if the space group is nonsymmorphic (it contains either a screw axis or glide-reflection plane), it will not be a subgroup. Because the symmetry operations of the point group leave the lattice points unchanged, the Wigner-Seitz cell is also invariant under the operations of the point group. The WignerSeitz cell is also space filling and is charge neutral. Thus the three properties that characterize a Wigner-Seitz cell are as follows: it is a space-filling polyhedron that has the symmetry of the point group of the lattice and is charge neutral.

In the case of diamond, there are two carbon atoms in the basis and the Wigner-Seitz cell as traditionally defined contains the same number of atoms as there are atoms in the basis, i.e., two carbon atoms. A Wigner-Seitz cell for an atom or, more properly, a Voronoi polyhedron, can be defined by analogy with the Wigner-Seitz cell for a lattice point. The region nearer to an atom at $\mathbf{R}_{I}$ than to an atom at $\mathbf{R}_{J}$ is the half-space

$$
D\left(\mathbf{R}_{I}, \mathbf{R}_{J}\right)=\left\{\mathbf{x}|| \mathbf{x}-\left.\mathbf{R}_{I}\right|^{2}<\left|\mathbf{x}-\mathbf{R}_{J}\right|^{2}\right\} .
$$

The intersection of all these half-spaces, i.e.,

$$
V\left(\mathbf{R}_{I}\right)=\bigcap_{J \neq I} D\left(\mathbf{R}_{I}, \mathbf{R}_{J}\right),
$$

defines the Voronoi polyhedron. Any point inside this cell is closer to the atom at $\mathbf{R}_{I}$ than to all other atoms (rather than to the lattice point as is the case for the traditional Wigner-Seitz cell). The Voronoi polyhedron, being the intersection of halfspaces, is a convex polyhedron.

Defined in this way, the Voronoi polyhedron possesses many properties similar to those of the standard WignerSeitz cell. It is a space-filling polyhedron and charge neutral. However, it is no longer necessarily invariant under the symmetry operations of the isogonal point group, but only under the operations of the largest point group that is a subgroup of the space group of the crystal. Further, the integral of the energy density over this volume is independent of any gauge and is equal to the total energy per atom.

If the basis atoms are not identical, for example as in GaAs, the Voronoi polyhedron will no longer be neutral as is desired. In this case, the Voronoi polyhedra is defined to be an additively weighted power Voronoi polyhedron. ${ }^{31}$ The additively weighted power Voronoi polyhedron or, more simply, the power Voronoi polyhedron extends naturally the definition of the Voronoi polyhedron from a lattice with a basis of identical atoms to a lattice with a basis of distinct atoms. In point of fact, the standard Wigner-Seitz cell, the Voronoi polyhedron for a lattice with a basis of identical atoms and the Voronoi polyhedron for a lattice with a basis of distinct atoms are all encompassed within the definition of the power Voronoi polyhedron.

The additively weighted power-distance of point $\mathbf{x}$ from an atom at $\mathbf{R}_{I}$ is

$$
d_{\mathrm{pw}}\left(\mathbf{x}, \mathbf{R}_{I} ; w_{I}\right)=\left|\mathbf{x}-\mathbf{R}_{I}\right|^{2}-w_{I}
$$

where $w_{I}$ is the weight associated with the atom at $\mathbf{R}_{I}$. With respect to the additively weighted power distance, the halfspace closer to an atom at $\mathbf{R}_{I}$ than to one at $\mathbf{R}_{J}$ is

$$
D\left(\mathbf{R}_{I}, \mathbf{R}_{J}\right)=\left\{\mathbf{x}|| \mathbf{x}-\left.\mathbf{R}_{I}\right|^{2}-w_{I}<\left|\mathbf{x}-\mathbf{R}_{J}\right|^{2}-w_{J}\right\}
$$

and the power Voronoi polyhedron is the intersection of the half-spaces, i.e.,

$$
V\left(\mathbf{R}_{I}\right)=\bigcap_{J \neq I} D\left(\mathbf{R}_{I}, \mathbf{R}_{J}\right) .
$$

The use of the additively weighted power distance is motivated by the observation that the planes defining the surfaces of the traditional Wigner-Seitz cell are defined through Eq. (A2) and that the additive weights rigidly translate the faces of the Wigner-Seitz cell, always maintaining a polyhedral shape. When $w_{I}=w=$ constant for a lattice without a basis, the standard Wigner-Seitz cell is obtained.

For a tetrahedrally coordinated compound semiconductor comprised of elements $A$ and $B$, the volume contained within the Voronoi polyhedron is a monotonic function of the difference of the weights $w_{A}$ and $w_{B}$. If charge-neutral polyhedra are used, the value of this difference is fixed by the requirement that the total electronic charge within the Voronoi polyhedron cell be equal to the ionic charge; if, instead, charged polyhedra are used, the difference can be chosen for convenience. In the case of $\mathrm{C}_{60}$, not all of the atoms are in symmetry-equivalent positions and charged Voronoi polyhedra must be used. Further, the space group is nonsymmorphic. Consequently, the largest point group that is a subgroup of the space group for solid $\mathrm{C}_{60}$ is not $T_{h}^{6}$, but $\overline{3}$ and the Voronoi polyhedra will have this symmetry.
${ }^{1}$ J. Tsao, Materials Fundamentals of Molecular Beam Epitaxy (Academic Press, Boston 1993).

${ }^{2}$ J. LaFemina, Phys. Rep. 16, 133 (1992).

${ }^{3}$ N. Chetty and R. M. Martin, Phys. Rev. B 45, 6074 (1992).

${ }^{4}$ N. Chetty and R. M. Martin, Phys. Rev. B 45, 6089 (1992).

${ }^{5}$ N. Chetty and R. M. Martin, Phys. Rev. B 44, 5568 (1991).
${ }^{6}$ C. Herring, in The Physics of Powder Metallurgy, edited by W. E. Kingston (American Society of Metals, Cleveland, Ohio, 1951), pp. 7 and 8 .

${ }^{7}$ W. Winterbottom, Acta Metall. 15, 303 (1967).

${ }^{8}$ J. Lee, H. Aaronson, and K. Russel, Surf. Sci. 51, 302 (1975).

${ }^{9}$ E. Arbel and J. Cahn, Surf. Sci. 51, 305 (1975). 
${ }^{10}$ G-X. Qian, R. Martin, and D. Chadi, Phys. Rev. B 38, 7649 (1988).

${ }^{11}$ There may also be other stoichiometries that will further limit the stability range, thus making this choice somewhat arbitrary, although conventional.

${ }^{12}$ This definition of the surface energy (at $T=0$ ) corresponds to Herring's specific surface (free) energy.

${ }^{13}$ M. Buerger, Elementary Crystallography (MIT Press, Cambridge, 1978).

${ }^{14}$ P. Hohenberg and W. Kohn, Phys. Rev. 136, 864B (1964).

${ }^{15}$ W. Kohn and L. Sham, Phys. Rev. 140, 1133A (1965).

${ }^{16}$ J. Slater, Phys. Rev. 51, 846 (1937).

${ }^{17}$ D. Cook, Schrödinger's Mechanics (World Scientific, Singapore, 1988).

${ }^{18}$ There is, in principle, no problem in choosing a different form for the kinetic energy density, provided it is used consistently. The choice serves to change the gauge.

${ }^{19}$ L. Föppl, Phys. Z. 15, 191 (1914).

${ }^{20}$ K. Critchlow, Order in Space: A Design Source Book (Viking, New York, 1969).

${ }^{21}$ W. Brostow, J.-P. Dusrault, and B. Fox, J. Comput. Phys. 29, 81 (1978).

${ }^{22}$ J. Finney, J. Comput. Phys. 32, 137 (1979).

${ }^{23}$ M. Tanemura, T. Ogawa, and N. Ogifa, J. Comput. Phys. 51, 191 (1983).

${ }^{24}$ N. Medvedev, J. Comput. Phys. 67, 223 (1986).

${ }^{25}$ R. Riedinger, M. Habar, P. Oelhafen, and H. Günterodt, J. Comput. Phys. 74, 61 (1988).

${ }^{26}$ M. P. Allen and D. J. Tildesley, Computer Simulation of Liquids (Clarendon Press, Oxford, 1987).

${ }^{27}$ O. Jepson and O. K. Anderson, Solid State Commun. 9, 1763 (1971)
${ }^{28}$ G. Lehmann and M. Taut, Phys. Status Solidi B 54, 469 (1972).

${ }^{29}$ H. Monkhorst and J. Pack, Phys. Rev. B 13, 5188 (1976).

${ }^{30}$ P. Blöchl, O. Jepsen, and O. K. Anderson, Phys. Rev. B 49, 16223 (1994).

${ }^{31}$ A. Okabe, B. Boots, and K. Sugihara, Spatial Tesselations: Concepts and Applications of Voronoi Diagrams (Wiley, Chichester, 1992).

${ }^{32}$ B. Joe, Adv. Eng. Softw. 13, 325 (1991).

${ }^{33}$ M. Abramowitz and I. Stegun, Handbook of Mathematical Functions (Dover, N.Y., 1972), p. 895.

${ }^{34}$ R. Car and M. Parrinello, Phys. Rev. Lett. 55, 2471 (1985).

${ }^{35}$ M. Payne, M. Teter, D. Allan, T. Arias, and J. Joannopoulos, Rev. Mod. Phys. 64, 1045 (1992).

${ }^{36}$ D. Remler and P. Madden, Mol. Phys. 70, 921 (1990).

${ }^{37}$ J. Perdew and A. Zunger, Phys. Rev. B 23, 5048 (1981).

${ }^{38}$ D. Ceperley and B. Alder, Phys. Rev. Lett. 45, 566 (1980).

${ }^{39}$ G. Bachelet, D. Hamann, and M. Schlüter, Phys. Rev. B 26, 4199 (1982).

${ }^{40}$ D. R. Hamann, M. Schlüter, and C. Chiang, Phys. Rev. Lett. 43, 1494 (1979).

${ }^{41}$ D. R. Hamann, Phys. Rev. B 40, 2980 (1989).

${ }^{42}$ G. Li and S. Rabii, 1992 (unpublished).

${ }^{43}$ L. Kleinman and D. Bylander, Phys. Rev. Lett. 48, 1425 (1982).

${ }^{44}$ S. Louie, S. Froyen, and M. L. Cohen, Phys. Rev. B 26, 1738 (1982).

${ }^{45}$ M. Buongiorno Nardelli, K. Rapcewicz, and J. Bernholc, Phys. Rev. B 55, R7323 (1997).

${ }^{46}$ Y. Hwang, B. Chen, P. Bogusławski, and J. Bernholc, in Covalent Ceramics II: Non-Oxides, edited by A. R. Barron, G. S. Fischman, M. A. Fury, A. F. Hepp, MRS Symposia Proceedings No. 327 (Materials Research Society, Pittsburgh, 1994), p. 293.

${ }^{47}$ C. J. Bradley and A. P. Cracknell, The Mathematical Theory of Symmetry in Solids (Oxford, Clarendon Press, 1972). 